\title{
Worst Observed Case Imputation Technique
}

National Cancer Institute

\section{Source}

National Cancer Institute. Worst Observed Case Imputation Technique. NCI Thesaurus.

Code C132343.

A data imputation technique that populates analysis values with the worst recorded outcome within a subject. 